\title{
土壤养分水平影响绢毛匍匐委陵菜匍匐茎生物量投资
}

\author{
张淑敏 于飞海 董 鸣* \\ (中国科学院植物研究所植被与环境变化国家重点实验室 北京 100093)
}

\begin{abstract}
摘 要 为研究匍匐茎草本植物对基质养分供应水平的生物量分配格局的可塑性,在一盆栽实验中对绢毛匍匐委 陵菜 (Potentilla reptans var. sericophylla) 进行了 8 种不同的养分处理。绢毛匍匐委陵菜植株生物量、匍匐茎数、分株 数以及匍匐茎节间长在中等养分条件下最大。随土壤养分的降低绢毛匍匐委陵菜对叶片和叶柄的生物量投资减 小, 而对根系的生物量投资增加。在中等养分条件下 绢毛匍匐委陵菜对匍匐茎的生物量投资倾向于最大, 而在更 高或更低的养分条件下倾向于减少。此生物量分配格局与 de Kroon 和 Schieving 的模型模拟结果相符合，结果表明 在中等资源水平下增加对匍匐茎的生物量投资是克隆植物增加资源获取的对策之一。
\end{abstract}

关键词 克隆植物 可塑性 生物量分配模型 生物量对匍匐茎的投资

\section{NUTRIENT AVAILABILITY AFFECTS BIOMASS ALLOCATION TO STOLONS IN POTENTILLA REPTANS VAR. SERICOPHYLLA}

\author{
ZHANG Shu-Min , YU Fei-Hai , and DONG Ming* \\ Key Laboratory of Vegetation and Environmental Change , Institute of Botany , Chinese Academy of Sciences , Beijing 100093 , China
}

\begin{abstract}
Aims Plasticity in biomass allocation of plants under different growing conditions is often assumed to be adaptive. Biomass allocation patterns in clonal plants are usually different from those in non-clonal plants due to clonal architecture and clonal morphology. de Kroon and Schieving (1991) published a model to predict the likely pattern of biomass allocation to spacers (i.e. , stolons or rhizomes) as resource supply varies. The model predicted that under moderate resource levels, clonal plants would allocate more biomass to spacers to efficiently utilize resources. The aim of this study is to experimentally test this prediction.

Methods In a greenhouse experiment, Potentilla reptans var. sericophylla plants were subjected to eight levels (i.e., N1 to N8 in decreasing order) of nutrient supply. N1 was equivalent to $600 \mathrm{~kg}$ nitrogen as $\mathrm{NH}_{4} \mathrm{NO}_{3}, 240 \mathrm{~kg}$ phosphorus as $\mathrm{NaH}_{2} \mathrm{PO}_{4}$ and $600 \mathrm{~kg} \mathrm{~K}$ as $\mathrm{KCl}$ per ha per year. $\mathrm{N} 2$ to $\mathrm{N} 8$ were $50 \%, 25 \%$, $12.5 \%, 6.25 \%, 3.13 \%, 1.57 \%$ and 0 of N1 , respectively. The experiment started on April 6,2003 and ended on June $3,2003$.

Important findings Potentilla reptans var. sericophylla had greater biomass, produced more stolons and had longer stolon internodes at moderate levels (i.e. , N3 and N4 treatments) of nutrient supply. Biomass allocation to different plant organs (roots, stolons, laminae and petioles) responded greatly to nutrient treatments. With decreasing nutrient supply, biomass allocation to laminae and petioles decreased, whereas that to roots increased. Biomass allocation to stolons tended to be the highest at moderate levels ( N3 , N4) of nutrient supply and became smaller at both lower and higher nutrient conditions. Patterns of biomass allocation to stolons in $P$. reptans var. sericophylla under different levels of nutrient supply agree with the model prediction of de Kroon and Schieving (1991), suggesting that allocating more biomass to spacers (i.e. , stolons or rhizomes) at moderate nutrient levels enables clonal plants to efficiently utilize resources.
\end{abstract}

Key words clonal plants , plasticity, biomass allocation model, biomass allocation to stolons

生物量分配格局的可塑性贯穿植物的整个生活 史 (Steams，1992），决定着植物在异质性环境中获取 资源的能力 ( Poorter et al . 1990)，从而影响其在群 落中的竞争力 (Grime, 1979 ; Tilman , 1988)。因此，
生物量分配格局对环境条件的可塑性具有重要的生 态适应意义(Grime，1979；Tilman，1988)。例如，在 光照资源比较低的情况下, 植物把生物量更多地分 配到地上茎、叶部分, 有利于对光资源的获取; 而在 
养分或水分资源贫㾑的情况下，植物把更多的生物 量分配到根部，从而利于对养分或水分资源的获取 ( van den Boogaard et al.,1997; Müller et al. , 2000 ; 于飞海等 ,2002 ; King , 2003)。

许多克隆植物由克隆分株和连接克隆分株的间 隔子构成, 具有独特的克隆构件性 (Slade \& Hucthings, 1987a ; de Kroon \& Knops , 1991 ; Dong \& de Kroon，1994)。一方面, 克隆植物独立分株具有与非 克隆植物相似的生物量分配格局 (Müller et al. , 2000 ; 董鸣等 ,2000; Liao et al. ,2003)。另一方面， 由于具有独特的克隆生活史性状特征, 如克隆整合、 克隆可塑性和克隆器官的存储功能等, 克隆植物的 生物分配格局又与非克隆植物不完全相同。例如， 克隆整合可以使得克隆分株的生物量分配格局对环 境因子的反应完全不同于非克隆植物的个体 (Stuefer , 1997 ; Liao et al. , 2003 ; Chu et al. ,2006)。再 者, 克隆植物可能通过间隔子长度、粗度和生物量分 配投资的可塑性选择性地放置克隆分株和储藏养 分 缓解资源的时空异质性对其生长和繁殖的不利 影响。例如, 欧活血丹 (Glechoma hederacea) 在低养 条件下, 匍匐茎节间增长而比匍匐节间茎重降低 (Slade \& Hucthings，1987b) ; 在土壤中氮的水平增加 时, 七瓣莲 (Trientalis europeaea) 对根状茎的总生物 量投资有增加的趋势(Dong et al . 1997)。

关于克隆植物的生物量分配格局研究有很多, 然而得到的结果却并不一致。这可能是由于植物种 受食行为” 的多样性所致, 也可能是资源水平梯度 设置不同造成的 (de Kroon \& Schieving，1991)。根据 实验获得的克隆植物分枝强度和分株间隔子对资源 水平的反应模式, de Kroon 和 Schieving (1991) 构建 了克隆植物在不同资源水平条件下生物量分配格局 的模型，并对其生物量分配格局的规律进行了模拟 和预测。该模型预测: 为实现对资源的最佳利用,在 中等资源水平条件下, 克隆植物对间隔子的生物量 分配将会最高。

为验证此模型的预测结果, 我们将来自同一个 克隆的绢毛匍匐委陵菜 (Potentilla reptans var. sericophylla) 分株种植于 8 种不同的基质养分环境中。通 过对植株各器官生物量的测定和对生物量分配格局 的分析, 探讨绢毛匍匐委陵菜在不同资源水平条件 下的生物量分配格局及其生态适应意义。

\section{1 材料和方法}

\section{1 物种}

绢毛匍匐委陵菜为菩薇科多年生草本植物。该 植物的幼苗(最初的地上枝系)生长到一定时期, 从 其直立茎基部节上产生侧向生长的一至数个匍匐 茎。当匍匐茎第一节间伸长后, 其顶芽则转向上生 长, 形成地上枝系, 匍匐茎第一节上的一侧芽激活形 成新的匍匐茎。同样, 新匍匐茎第一节间伸长后, 其 顶芽也转向上生长, 形成地上枝系, 新匍匐茎第一节 上的一个侧芽激活, 形成新的匍匐茎。这一过程不 断进行 就产生了一条相当长的匍司茎。所有地上 枝系都能从由多个极度缩短的节间构成的茎基上产 生多个匍匐茎。每一地上枝系都产生不定根, 成为 分株（张淑敏等, 2000)。匍匐茎节间长约 $10 \sim 20$ $\mathrm{cm}$ 将分株连接起来。匍匐茎能维持一个生长季节 不断裂。因此, 在自然生境中, 在生长季节, 通常可 以观察到该植物合轴分枝的地面匍匐茎网络系统。 该植物生长在暖温带和亚热带的山坡和湿地生境 中。在河北、内蒙古、山西、陕西、甘肃、山东、江苏、 浙江、河南、四川和云南等地均有分布 (贺士元等, 1997)。

\section{2 实验设计}

实验植物材料绢毛匍匐委陵菜于 2002 年采自 北京东灵山中国科学院植物研究所北京森林定位研 究站。采来的一个克隆基株培养一个夏天后, 形成 大量分株，次年返青后根据分株的生长状况，以 8 个 分株为一组共分成 8 组进行养分实验。所选分株大 小相近，以保证实验用植株材料的一致性。

实验设 $\mathrm{N} 1$ 至 $\mathrm{N} 8$ 共 8 个养分水平, 从 $\mathrm{N} 1$ 到 $\mathrm{N} 8$ 养分依次递减。养分以 $\mathrm{NH}_{4} \mathrm{NO}_{3} 、 \mathrm{NaH}_{2} \mathrm{PO}_{4}$ 和 $\mathrm{KCl}$ 的 混合营养液 (水溶液)的形式施加。每个处理每两个 星期施入体积相同 (10 ml) 但浓度不同的营养液。 $\mathrm{N} 1$ 营养液的浓度为 $20.6 \mathrm{~g} \mathrm{~L}^{-1} \mathrm{NH}_{4} \mathrm{NO}_{3} 、 14.08 \mathrm{~g}$ $\mathrm{L}^{-1} \mathrm{NaH}_{2} \mathrm{PO}_{4}$ 和 $14.2 \mathrm{~g} \mathrm{~L}^{-1} \mathrm{KCl}$, 在整个实验过程中 的施加总量相当于每年每公顷添加 $600 \mathrm{~kg} \mathrm{~N} 、 240$ $\mathrm{kg}$ P和 $600 \mathrm{~kg} \mathrm{~K} 。 \mathrm{~N} 2$ 至 $\mathrm{N} 8$ 营养液的浓度为 $\mathrm{N} 1$ 的 $50 \% 、 25 \% 、 12.5 \% 、 6.25 \% 、 3.13 \% 、 1.57 \%$ 和 0 。每 个处理设 8 个重复。

实验植株种植在直径 $10 \mathrm{~cm}$ 、高 $7.5 \mathrm{~cm}$, 盛有河 沙的 PVC 塑料种植钵内。每钵 1 个分株。实验期间 适时适量施水, 使水分条件处于满足植物生长需要 的非限制状态。实验期间所产生的新分株在新增加 的种植钵内扎根, 生长条件与母株相同。

实验在中国科学院植物研究所北京植物园内温 室内进行，2003 年 4 月 6 日开始，6月 3 日收获。实 验期间温室的温度维持在 $17 \sim 25{ }^{\circ} \mathrm{C}$, 湿度约 $80 \%$ 。 


\section{3 测量与数据分析}

收获后分别测定实验植株的分株数、匍匐茎数 和匍匐茎节间长。然后分为根、匍匐茎、叶片和叶 柄。各部分植物材料置于烘箱内在 $75{ }^{\circ} \mathrm{C}$ 下烘干至 恒重, 冷却后在精度为 $0.0001 \mathrm{~g}$ 的天平上称量。应 用 SAS 的 One-Way ANOVA 和 Duncan 多重比较方法 进行数据分析(SAS ,1997)。

\section{2 实验结果}

\section{1 克隆生长和形态}
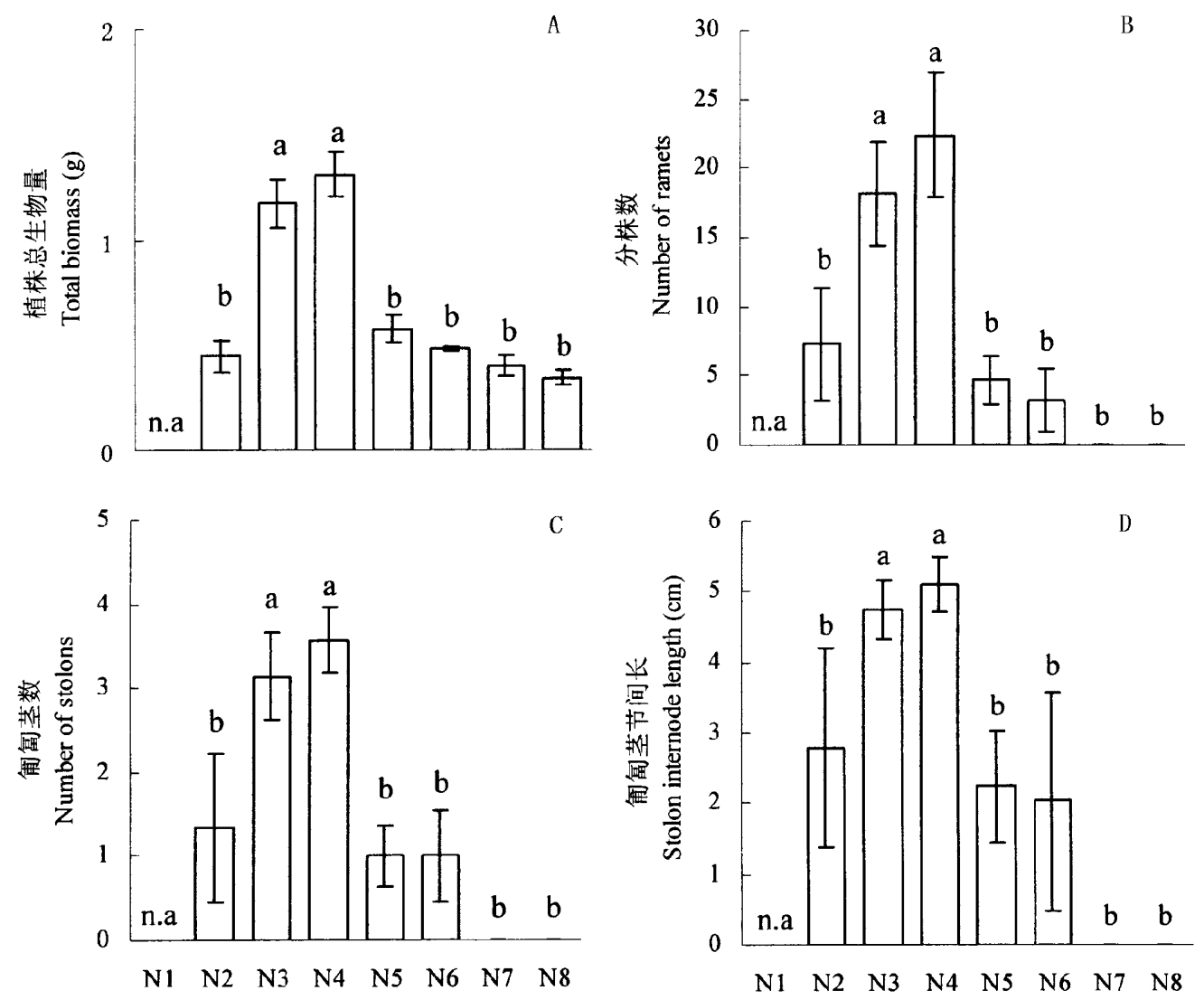

养分处理

Nutrien L treatments

图 1 绢毛匍匐委陵菜 (Potentilla reptans var. sericophylla) 基株生物量 $(\mathrm{A})$ 、分株数 $(\mathrm{B})$ 、匍匐茎数 $(\mathrm{C})$ 和匍匐茎节间长 (D)对土壤养分水平的反应 Fig. 1 Total biomass of genet (A), number of ramets (B), number of stolons (C) and stolon internode length (D) of Potentilla reptans var. sericophylla plants grown under eight nutrient treatments from N1 (the highest nutrient condition) to N8 (the lowest nutrient condition).

图中垂直线表示标准误差 相同字母表示在 $p=0.05$ 下差异不显著 Vertical line indicates standard error $( \pm S E)$. Bars sharing the same letters are not different at $p=0.05 \mathrm{~N} 1: 600 \mathrm{~kg} \mathrm{~N} \mathrm{hm}^{-2} \mathrm{a}^{-1}, 240 \mathrm{~kg} \mathrm{P} \mathrm{hm}^{-2} \mathrm{a}^{-1}, 600 \mathrm{~kg} \mathrm{~K} \mathrm{hm}^{-2} \mathrm{a}^{-1} \mathrm{~N} 2: 300 \mathrm{~kg} \mathrm{~N} \mathrm{hm} \mathrm{h} \mathrm{a}^{-1}, 120 \mathrm{~kg} \mathrm{P} \mathrm{hm}^{-2} \mathrm{a}^{-1}, 300 \mathrm{~kg}^{-2}$ $\mathrm{K} \mathrm{hm}^{-2} \mathrm{a}^{-1} \mathrm{~N} 3: 150 \mathrm{~kg} \mathrm{~N} \mathrm{hm}^{-2} \mathrm{a}^{-1}, 60 \mathrm{~kg} \mathrm{P} \mathrm{hm}^{-2} \mathrm{a}^{-1}, 150 \mathrm{~kg} \mathrm{~K} \mathrm{hm}^{-2} \mathrm{a}^{-1} \quad \mathrm{~N} 4: 75 \mathrm{~kg} \mathrm{~N} \mathrm{hm}^{-2} \mathrm{a}^{-1}, 30 \mathrm{~kg} \mathrm{P} \mathrm{hm}^{-2} \mathrm{a}^{-1}, 75 \mathrm{~kg} \mathrm{~K} \mathrm{hm}^{-2} \mathrm{a}^{-1}$ $\mathrm{N} 5: 37.5 \mathrm{~kg} \mathrm{~N} \mathrm{hm}^{-2} \mathrm{a}^{-1}, 15 \mathrm{kgP} \mathrm{hm}^{-2} \mathrm{a}^{-1}, 37.5 \mathrm{~kg} \mathrm{~K} \mathrm{hm}^{-2} \mathrm{a}^{-1} \mathrm{~N} 6: 18.8 \mathrm{~kg} \mathrm{~N} \mathrm{hm}^{-2} \mathrm{a}^{-1}, 7.5 \mathrm{~kg} \mathrm{P} \mathrm{hm}^{-2} \mathrm{a}^{-1}, 18.8 \mathrm{~kg} \mathrm{~K} \mathrm{hm}^{-2} \mathrm{a}^{-1} \mathrm{~N}$ : $9.4 \mathrm{~kg} \mathrm{~N} \mathrm{hm}^{-2} \mathrm{a}^{-1}, 3.75 \mathrm{~kg} \mathrm{P} \mathrm{hm}^{-2} \mathrm{a}^{-1}, 9.4 \mathrm{~kg} \mathrm{~K} \mathrm{hm}^{-2} \mathrm{a}^{-1} \mathrm{N8}: 4.7 \mathrm{~kg} \mathrm{~N} \mathrm{hm}^{-2} \mathrm{a}^{-1}, 1.88 \mathrm{~kg} \mathrm{P} \mathrm{hm}^{-2} \mathrm{a}^{-1}, 4.7 \mathrm{~kg} \mathrm{~K} \mathrm{hm}^{-2} \mathrm{a}^{-1}$

2.2 生物量分配

叶片、叶柄、根系和匍匐茎各个器官的生物量投
资表现出各自不同的趋势(图 2)。对根系的生物量 投资, 7 个养分处理形成两个梯度, N2、N3 和 N4 - 
组数值接近, 而且该组内所有处理的值均低于 N5、 N6、N7 和 N8 一组的 4 个处理, 两组的差异达到显著 水平 $(p<0.05)$ 。植株对叶片的生物量投资也基本 呈现两个梯度, 但是与对根系生物投资正好呈现出 相反的趋势, 在 $p=0.05$ 的水平上, N2、N3、N4 显著 高于 N5、N7 和 N8。对叶柄生物量的投资下 N2 下最
高, 而从 $\mathrm{N} 2$ 到 $\mathrm{N} 8$ 逐渐降低; 在 $p=0.05$ 水平上, $\mathrm{N} 7$ 和 N8 与处理 N3 和 N4 的差异达到显著水平。对匍 匐茎的生物量投资在 $\mathrm{N} 4$ 下最高而在 $\mathrm{N} 7$ 和 $\mathrm{N} 8$ 下最 低; $\mathrm{N} 4$ 与 $\mathrm{N} 6 、 \mathrm{~N} 7 、 \mathrm{~N} 8$ 在 $p=0.05$ 的显著性水平下差 异显著, 而 N2、N3、N4 和 N5 之间匍匐茎生物量投资 差异不显著。
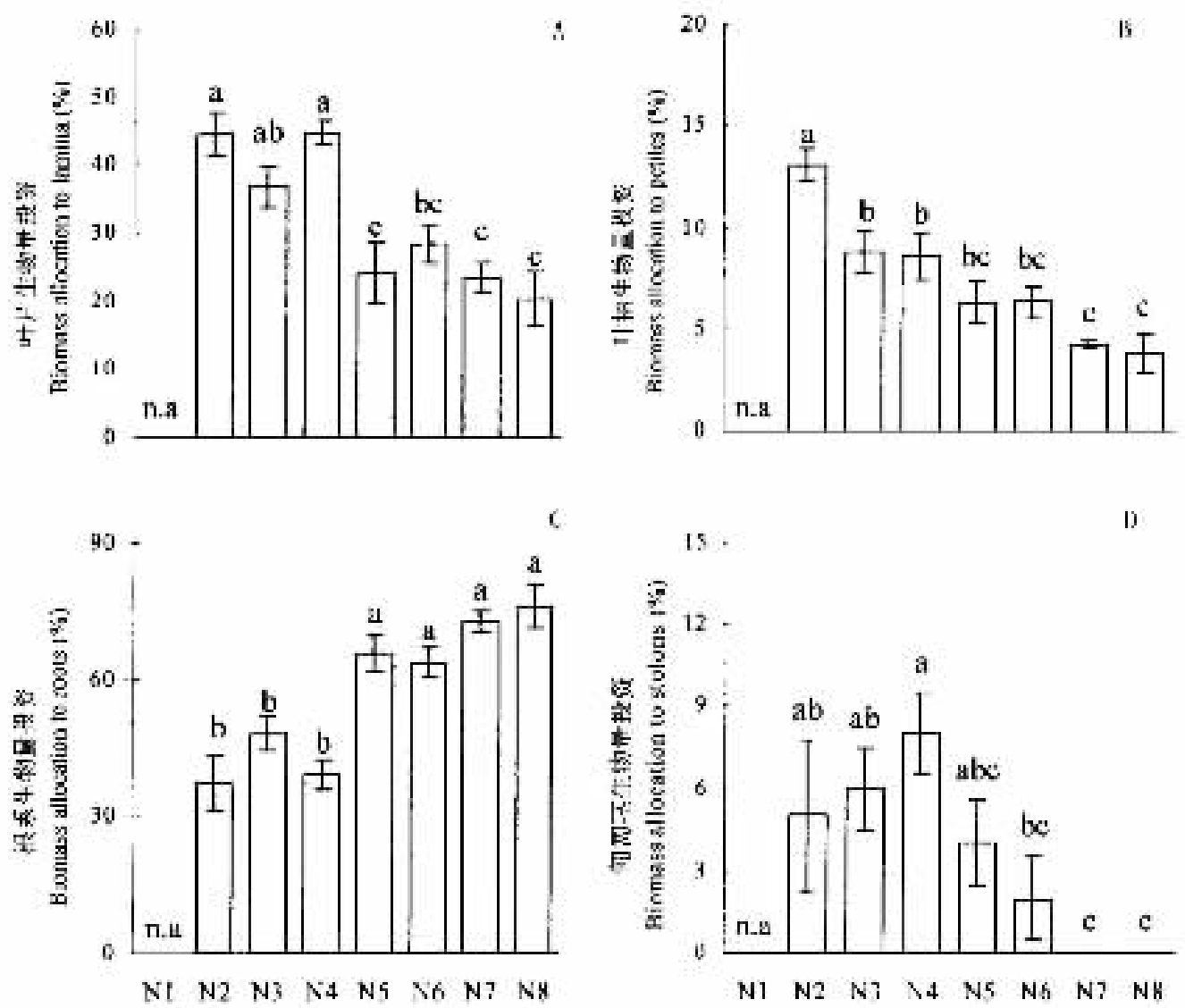

15.

D)

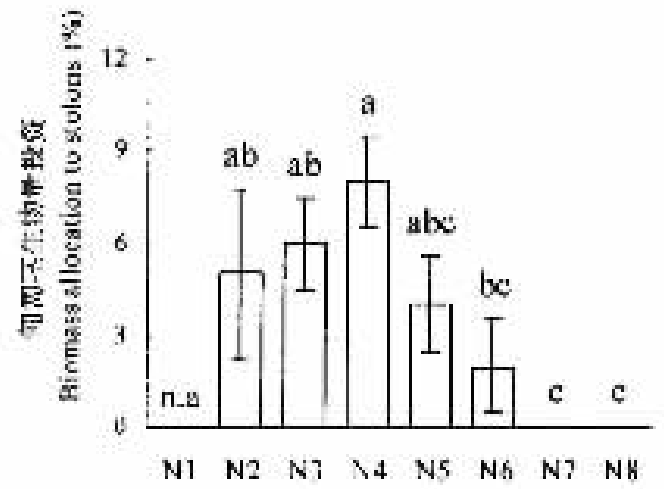

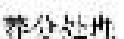

Sutroeat, Trentrients

图 2 绢毛匍匐委陵菜 (Potentilla reptans var. sericophylla) 的叶片生物量投资 $(\mathrm{A}) 、$ 、叶柄生物量投资 $(\mathrm{B}) 、$ 根系生物量投资 $(\mathrm{C})$ 和 匍匐茎生物量投资 $(\mathrm{D})$ 对土壤养分水平的反应

Fig.2 Biomass allocation to blades (A), biomass allocation to petioles (B), biomass allocation to roots and biomass allocation to stolons (D) of Potentilla reptans var. sericophylla plants grown under eight nutrient treatment from N1 (the highest nutrient condition) to N8 (the lowest nutrient condition) 图注同图 1 Notes see Fig. 1

\section{3 讨 论}

克隆可塑性是克隆植物适应异质性生境的重要 对策之一, 体现在克隆生长、克隆形态以及对克隆器 官生物量投资等方面 (Slade \& Hucthings，1987b； Dong et al., 1997 ; Yu et al .,2004)。在本研究中, 以 N4 处理设置为植物生长较适宜的养分条件, 并 以此为基础依次增加和减少土壤养分的施用。研究
中发现, 在养分适中的处理 N3 和 N4 中, 基株总生 物量最高。这表明了适度的养分能促进植物生长, 过低或者过高能够抑制植物生长, 养分过高甚至发 生盐害导致植株死亡 (于飞海等, 2002)。同样, 在 养分适中条件下, 克隆生长得到促进 绢毛匍匐委陵 菜形成更多匍匐茎。绢毛匍匐委陵菜匍匐茎数这种 对土壤基质养分梯度的反应格局与大多数克隆植物 的反应格局相一致, 如蛇莓 (Duchesnea indica) 以及 
Amphibromus scabrivalvis 等 (Cheplick \& Grandstaff, 1995 ; 董鸣等, 2000)。养分适宜的条件下, 克隆植 物产生更多地匍匐茎可以使克隆分株占据更多的空 间 获取更多的环境资源。

生物量分配格局可塑性具有重要的生态适应意 义(Dong \& de Kroon , 1994 ; Hutchings , 1997)。本研 究中发现 绢毛匍匐委陵菜的匍匐茎生物量投资对 土壤养分变化发生了可塑性反应, 表现出一种趋势, 即在养分适中的条件下, 植株对匍匐茎的生物量投 资最高。而养分过高或过低都不利于匍匐茎生长, 甚至在低养分条件下实验植株没有匍匐茎生成。董 鸣等 (2000) 对蛇莓的研究表明在中等强度的养分 条件下植株对匍匐茎的投资最高, 与本实验结果一 致。同时, 本实验的结果符合 de Kroon 和 Schieving (1991) 的模型预测。这表明，在适宜的条件下克隆 植物增加对匍匐茎的投资, 可以在储存养分的同时 增加分枝强度, 使克隆植物有可能占据更广阔的生 存空间。这种匍匐茎生物量分配对基质养分的可塑 性是克隆植物获取资源、适应异质性环境的重要对 策之一, 是克隆植物 受食行为”的一种表现形式。 然而，Pelling (1994;引自 Hutchings，1997)的实验却 发现，在低养分的条件下 Glechoma hederaea 对匍匐 茎的生物量投资倾向最大。这种差异表明克隆植物 的受食行为是多样的。

绢毛匍匐委陵菜的其它器官生物量分配对土壤 养分也发生了可塑性反应。在基质养分逐渐增加的 情况下 绢毛匍匐委陵菜对根系生物量投资逐渐增 加，对叶片和叶柄的生物量投资随着养分含量的降 低而降低。绢毛匍匐委陵菜对根系的投资格局与活 血丹 (Glechoma hederacea) 和草莓 (Fragaria chiloensis) 在基质养分贫痛的生境中增加对根系的投资是相同

的 (Slade \& Hutchings, 1987a ; Alpert \& Mooney, 1991)。Müller 等 (2000) 对野外 27 种植物 (其中绝大 部分为克隆植物) 的研究也发现了类似的规律。在 低养环境中植物增加对根系的投资, 可以提高植物 获取这种限制性资源的能力（Bradshaw，1965; Chapin , 1980 ; Wilson \& Thompson, 1989 ; Gedroc et $a l$, , 1996)。而叶片是植株获取光资源的主要器官, 在温室中, 光不是制约条件, 减少对叶片的投资, 就 可能将这部分资源投向根系, 这可能是植株适应异 质性环境的重要对策。

本试验中，绢毛匍匐委陵菜匍匐茎节间长在中 等养分水平下最长。以往对匍匐茎节间长度对土壤 养分可塑性的反应并没有统一定论。有研究表明在
土壤养分水平较高的条件下, 唇形科的活血丹 ( Glechoma hederacea) 匍匐茎节间缩短, 使克隆分株尽可 能多地生长在有利的斑块中 ( de Kroon \& Knops , 1990) 然而蓄薇科的蛇莓 (董鸣等，2000) 和草莓 (Alpert \& Mooney，1991) 以及毛莨科的匍枝毛莨 (Ranunculus repens) (Lovett-Doust，1987) 在不同土壤 养分梯度下, 匍匐茎节间长没有发生改变, 对土壤养 分没有发生可塑性反应。本实验绢毛匍匐委陵菜匍 匐茎节间对土壤基质养分的可塑性发生了反应，在 养分适中的条件下增长，与对中国狗牙根( Cynodon dactylon)的研究结果一致 (Dong \& de Kroon, 1994)。 这可能是在养分适中的条件下匍匐茎节间的伸长， 有利于植物扩展更大空间, 获取更多资源, 对于克隆 植物而言，不同物种的反应不完全一致，表明“受食 行为”的多样性，生态适应的多样性。

\section{参 考 文 献}

Alpert P, Mooney HA (1991). Nitrogen sharing among ramets increases clonal growth in Fragaria chiloensis. Ecology, 72, $69-$ 80 .

Bradshaw AD ( 1965$)$. Evolutionary significance of phenotypic in plants. Advances in Genetics, 13, $115-155$.

Chapin FS (1980). The mineral nutrition of wild plants. Annual Review of Ecology and Systematics, 11, 233-260.

Cheplick GP, Grandstaff K (1995). Genotypic variation and plasticity of clonal growth in relation to nutrient availability in Amphibromus scabrivalvis. Journal of Ecology, 83, $459-468$.

Chu Y, Yu FH, Dong M (2006). Clonal plasticity in response to reciprocal patchness of light and nutrients in the stoloniferous herb Glechoma longituba L. Journal of Integrative Plant Biology, 48, $400-408$.

de Kroon H, Knops J (1990). Habitats exploration through morphological plasticity in two chalk grassland perennials. Oikos, $59,39-49$.

de Kroon H, Schieving F (1991). Resource allocation patterns as a function of clonal morphology: a general model applied to a foraging clonal plant. Journal of Ecology, 79, 519-530.

Dong M, de Kroon H (1994). Plasticity in morphology and biomass allocation in Cynodon dactylon, a grass species forming stolons and rhizomes. Oikos, 70, 99-106.

Dong M, During H, Werger MJA (1997). Clonal plasticity in response to nutrient availability in the pseudoannual herb, Trientalis europaea L. Plant Ecology, 131, 233-239.

Dong M (董鸣), Zhang SM (张淑敏), Chen YF (陈玉福) (2000). Clonal plasticity in response to nutrient availability in the stoloniferous herb Duchesnea indica. Acta Botanica Sinica (植物学报), 42, 518-522. (in Chinese with English abstract)

Gedroc JJ, McConnaghay KDM, Coleman JS (1996). Plasticity in 
root/shoot partitioning, optimal, ontogenetic, or both? Functional Ecology, 10, 44 - 50.

Grime JP (1979) . Plant Strategies and Vegetation Processes. Wiley, Chichester.

He SY (贺士元), Xing QH (邢其华), Yin ZT (尹祖堂), Jiang XP (江先普) (1997). Flora of Beijing (北京植物志). Vol. 1. Beijing Publishing House, Beijing. (in Chinese)

Hutchings MJ (1997). Resource allocation patterns in clonal herbs and their consequences for growth. In: Bazzaz FA, Grace J eds. Plant Resource Allocation, Chapter 7. Academic Press, New York, 161 - 186.

King DA (2003). Allocation of above-ground growth is related to light in temperate deciduous saplings. Functional Ecology, 17, $482-488$.

Liao MJ, Yu FH, Song MH, Zhang SM, Zhang JZ, Dong M (2003). Plasticity in $\mathrm{R} / \mathrm{S}$ ratio, morphology and fitness-related traits in response to reciprocal patchiness of light and nutrients in the stoloniferous herb, Glechoma longituba L. Acta Oecologica, $24,231-239$.

Lovett-Doust L (1987). Population dynamic and local specialization in a clonal perennial (Runuculus repens). III . Response to light and nutrient supply. Journal of Ecology, 75, 555 - 568 .

Müller I, Schmid B, Weiner J (2000). The effects of nutrient availability on biomass allocation patterns in 27 species of herbaceous plants. Perspectives in Plant Ecology, Evolution and Systematics, 3/2, 115 - 127 .

Pelling V (1994). Factory Influencing the Vegetation Growth of Clonal Perennial Species in the Family Lamiaceae. PhD dissertation, University of Sussex, Sussex, UK.

Poorter H, Remkes C, Lambers H (1990) . Carbon and nitrogen economy of twenty-four wild species differing in relative growth rate. Plant Physiology, 94, $621-627$.

SAS/STAT (1997) . Guide for personal computer versions. SAS In- stitute Inc., Gary, USA.

Slade AJ, Hutchings MJ (1987a). An analysis of the costs and benefits of physiological integration between ramets in the clonal perennial herb Glechoma hederacea. Oecologia, 73, 425-431.

Slade AJ, Hutchings MJ (1987b). The effects of nutrient availability on foraging in the clonal herb Glechoma hederacea. Journal of Ecology, 75, 98 - 112 .

Steams SC (1992). The Evolution of Life Histories. Oxford University Press, Oxford.

Stuefer JF (1997) . Division of Labour in Clonal Plants? On the Response of Stoloniferous Herb to Environmental Heterogeneity. $\mathrm{PhD}$ dissertation, Utrecht University Press, The Netherlands.

Tilman D (1988). Plant Strategies and the Dynamics and Structure of Plant Communities. Princeton University Press, Princeton.

van den Boogaard R, Alewijnse D, Veneklaas EJ, Lambers H (1997). Growth and water-use efficiency of 10 Triticum aestivum cultivars at different water availability in relation to biomass allocation. Plant, Cell and Environment, 20, 200-210.

Wilson AM, Thompson K (1989) . A comparative study of reproductive allocation in 40 British grasses. Functional Ecology, 3, $297-302$.

Yu FH, Dong M, Zhang CY, Zhang SM (2002). Phenotypic plasticity in response to salinity gradient in a stoloniferous herb Halerpestes ruthenica. Acta Phytoecologica Sinica (植物生态学报), $26,140-148$

Yu FH, Dong M, Krusi B (2004). Clonal integration helps Psammochloa villosa survive sand burial in an inland dune. New Phytologist, 162, $697-704$.

Zhang SM (张淑敏), Chen YF (陈玉福), Dong M (董鸣) (2000) . Clonal plasticity in response to partial neutral shading in the stoloniferous herb Potentilla reptans var. sericophylla. Acta Botanica Sinica (植物学报), 42,89-94. (in Chinese with English abstract) 